\title{
Criminologie
}

\section{La question du secret professionnel dans l'intervention clinique auprès des délinquants adultes}

\section{Richard Lusignan}

Volume 26, numéro 2, 1993

La criminologie comparée. Hommage à Denis Szabo

URI : https://id.erudit.org/iderudit/017343ar

DOI : https://doi.org/10.7202/017343ar

Aller au sommaire du numéro

Éditeur(s)

Les Presses de l'Université de Montréal

ISSN

0316-0041 (imprimé)

1492-1367 (numérique)

Découvrir la revue

Citer cet article

Lusignan, R. (1993). La question du secret professionnel dans l'intervention clinique auprès des délinquants adultes. Criminologie, 26(2), 137-153.

https://doi.org/10.7202/017343ar
Résumé de l'article

The conditions and the rules of practice in clinical settings limit the client's right to confidentiality. These limits are reviewed in the case of criminologists : some limits are legal while others refer to the context, the expectations and the reticences shared by the speakers in presence.

In regard to past crimes and s elf-incrimination, these questions raise fundamental issues both to the level of ethics and administration of the penal justice. Besides the latter, is there a place for moral justice in our social control system? 
The conditions and the rules of practice in clinical settings limit the client's right to confidentiality. These limits are reviewed in the case of criminologists: some limits are legal while others refer to the context, the expectations and the reticences shared by the speakers in presence.

In regard to past crimes and self-incrimination, these questions raise fundamental issues both to the level of ethics and administration of the penal justice. Besides the latter, is there a place for moral justice in our social control system?

\section{INTRODUCTION}

Le droit au secret professionnel est un droit inscrit en toutes lettres dans la Charte des droits et libertés de la personne du Québec (article 9). Par définition, celui-ci suppose que toute personne possédant des renseignements confidentiels, révélés en raison de la profession qu'elle exerce, ne peut les divulguer à moins de n'y être autorisé par celui qui a fait ces confidences, à moins aussi qu'elles ne constituent une exception spécifique prévue par une loi (v.g. Loi de la protection de la jeunesse). Notons également qu'une confidence qui aurait trait à la préparation d'un acte illicite ne tombe pas sous le coup du secret professionnel.

Malgré ce qui précède, sur le plan strictement légal, la reconnaissance provinciale de ce droit soulève plusieurs questions de grand intérêt; notamment au niveau de la préséance des juridictions. En effet, le secret professionnel n'est reconnu ni par le common law ni par la Charte canadienne des droits et libertés; ceci bien que cette charte ne soit pas limitative quant à l'existence d'autres droits au Canada.

Le cas spécifique de l'intervention clinique auprès des délinquants adultes implique donc un paradoxe : celui du droit du client au secret professionnel et de la «contraignabilité » des cliniciens professionnels en cour criminelle.

Au Canada, des milliers de personnes se trouvent détenues en vertu de l'application du code criminel. Un certain nombre de celles-ci sont hospitalisêes dans des établissements à vocation médico-légale où on les incite à participer à leur traitement. Ce faisant ces personnes sont invitées à discuter avec les intervenants l'ensemble de leurs agissements délinquants réels. Le

1. L'auteur est étudiant au doctorat et criminologue a l'Institut Philippe-Pinel de Montréal, 10905 est, boul. Henri-Bourassa. Montréal, Québec, H1C 1 H1. 
but évident est d'optimiser les possibilités de réussite des interventions cliniques ; cependant, en agissant de la sorte, ces personnes s'exposent à la possibilité de voir leur confident professionnel contraint de témoigner en cour criminelle concernant leurs antécédents.

Devant un refus de collaborer avec les intervenants, les possibilités d'amélioration des conditions psychologiques de ces individus s'amenuisent. La Cour suprême du Canada a été saisie de ce dilemme en 1989; jusqu'à présent, aucun jugement n'est venu trancher la question.

Dans un premier temps, nous allons baliser le sujet de cet article en prenant comme référence le modèle psychiatrique. Cette approche identifiera les origines et les échelles remises en question du modèle clinique médical face à la confidentialité.

Dans un second temps, nous analyserons la problématique de la confidentialité dans l'intervention clinique criminologique auprès de détenus adultes. Afin de mieux saisir l'état de cette question, nous procéderons à la comparaison entre la confidentialité offerte en milieu pénitentiaire et celle disponible en milieu hospitalier afin d'en distinguer les diverses composantes.

\section{LA CONFIDENTIALITÉ ET LE MODÈLE MÉDICAL}

Historiquement, la gestion de la confidentialité apparaît étroitement liée à l'émergence et à la popularité du modèle médical hippocratique. Le serment d'Hippocrate remonte au IV' ${ }^{\mathbf{s}}$ iècle avant Jésus Christ. Le serment lui-même se compose de deux engagements différents (Moore (1978) et Appelbaum (1984)). Dans le premier, le médecin s'engage, d'une part, à honorer son maître comme s'il était un parent et, d'autre part, à enseigner aux fils de son maître, la transmission de son savoir aux générations suivantes étant le seul moyen de s'acquitter de sa dette à l'endroit de celui qui lui a enseigné.

Le second engagement consiste en une série de règles relatives aux transactions avec les patients: de manière générale, veiller à leur soin et leur mieux-être, s'abstenir de contact sexuel, ne pas pratiquer de chirurgie et protéger la confidentialité des informations obtenues. Notons qu'il s'agit ici d'une confidentialité absolue, puisque les dires des clients revêtaient une valeur de secrets sacrés.

Appelbaum (1984) souligne que cet intérêt envers la confidentialité peut relever de la coexistence de deux types de motivations : l'une déontologique, l'autre historique et pratique. Ainsi, l'on peut penser qu'il s'agit, chez les médecins, de la reconnaissance morale de protéger les confidences dont ils ont eux-mêmes suscité et encouragé l'expression chez le client. Une autre argumentation plausible tient à ce que ce serment encourageait les patients de la Grèce antique à chercher un traitement et à se confier 
auprès d'un médecin ambulant qu'ils rencontraient vraisemblablement pour la première fois. À l'époque, il existait un climat de compétition entre les diverses sectes de médecins itinérants et le fait de se mériter la confiance du public présentait un avantage économique évident.

Selon Moore (1978) et Del Rio (1980 et 1985), le serment d'Hippocrate connut un tel retentissement qu'il fut traduit en hébreu, en arabe et en latin. Toujours selon ces auteurs, le serment est à la base de l'éthique médicale occidentale et on peut en voir l'influence dans des transcriptions plus modernes telles que le code d'éthique médicale de Percival (1794), celui de l'American Medical Association (1847) et de l'American Psychiatric Association (1970). Ajoutons que l'esprit de ce serment fonde aussi les codes éthiques et déontologiques des autres professions cliniques ou psychothérapeutiques s'inspirant du modèle médical.

La confidentialité telle que définie dans le serment hippocratique a connu des modifications conceptuelles majeures au fil des siecles. L'obligation sacrée de ne révéler aucune des confidences du client n'a plus cours aujourd'hui. Selon l'expression de Freedman (1980), certains de ces changements étaient nécessaires, d'autres inévitables alors qu'un certain nombre demeurent injustifiés.

Appelbaum (1984) explique ces changements par l'évolution du rapport existant entre le droit à l'intimité des clients et divers autres droits pouvant aussi se formuler en termes éthiques. À titre d'exemple, les confidences d'un individu peuvent ainsi être rapportées lors de la révision d'une ordonnance de cure fermée, afin de protéger une tierce partie d'un comportement violent, en rapport avec certaines lois à déclaration obligatoire ( $v . g$. la Loi de la protection de la jeunesse), etc.

Au cours des dernières décennies, des bris spectaculaires de confidentialité ont alarmé la communauté psychiatrique américaine. La divulgation des traitements psychiatriques (hospitalisations et sismothérapie) subis par le candidat démocrate au poste de vice-président en 1972; le cambriolage du cabinet du psychiatre de Daniel Ellsberg qui faisait alors face à des accusations de trahison demeurent présents à l'esprit de plusieurs psychiatres.

Les inquiétudes soulevées par ces intrusions dans la relation soignant/soigné touchent plusieurs aspects plus quotidiens : la tenue des dossiers, l'entreposage et la destruction du matériel clinique écrit, les modalités de diffusion des informations médicales auprès des forces de l'ordre, des compagnies d'assurances, etc. (Freedman, 1980). Le développement des banques de données informatisées et l'échange d'informations qu'elles permettent inquiètent également plusieurs auteurs: Winslade (1989), entre autres, note que, jusqu'à présent, les efforts pour procurer une protection légale efficace aux données confidentielles n'ont été que partiellement couronnés de succès. 
Par ailleurs, les médias s'intéressent ponctuellement à l'activité des psychiatres, que ce soit à la faveur d'un procès qui fait la manchette des journaux ou à l'occasion de l'hospitalisation d'une personnalité publique ou notoire. Dans ce contexte, les médias peuvent tenter d'obtenir des informations (cliniques ou autres) en s'adressant aux médecins ou aux employés de ces établissements. L'opposition entre le droit à l'intimité et celui de savoir s'illustre également dans le domaine de la recherche par l'opposition entre le droit à l'intimité des individus et le besoin de savoir des chercheurs (Freedman, 1980).

Au niveau plus spécifique des interventions cliniques, il nous faut citer le cas des thérapies de groupe et de couple, où les participants ne sont liés par aucune obligation éthique à la confidentialité des éléments qui s'y révèlent. Redlich et Mollica (1976) rappellent avec justesse que les problèmes de confidentialité sont inhérents à ces formes d'approches : même si l'animateur du groupe précise l'importance de limiter les fuites, il ne possède pas de moyens de contrôle sur le comportement des autres participants.

L'expertise médico-légale dans le but d'établir l'aptitude, la responsabilité criminelle du prévenu ou les mesures présentencielles appropriées présente un problème particulier, car, contrairement aux exemples précédents, la transgression de la règle hippocratique de la confidentialité absolue y est implicite. Le fait d'être retenu par une tierce partie ne réduit en rien la responsabilité de l'expert à l'égard de la confidentialité : mandaté pour faire connaître la personne évaluée aux autres parties légalement concernées (procureur de la défense ou de la Couronne, juge ou jurés), l'expert ne peut se soustraire à l'évocation des dires ou des agissements rapportés par le client. Par ailleurs, le client devrait toujours être informé du but de l'évaluation effectuée et de l'étendue de la confidentialité établie par l'expert, la sélection des données transmises devant s'effectuer selon la pertinence des informations recueillies par rapport à la situation judiciarisée (Appelbaum, 1984).

Enfin demeurent les situations liées au suivi de personnes violentes. Ici, la question de la confidentialité se présente principalement de deux façons, selon qu'il s'agisse d'un acte violent projeté ou d'un fait accompli. Dans la première situation, le devoir d'avertir, sinon de protéger, les tierces parties en tant que victimes possibles d'un individu potentiellement dangereux peuvent amener le psychiatre traitant à dénoncer son client aux autorités policières ${ }^{2}$

2. Les jugements américains de première instance et d'appel prononcés dans l'affaire Tarasoff (1976) ont successivement pris l'une et l'autre de ces formes. Ce jugement se rapporte au cas d'un psychologue californien a qui un client, dont il assumait le suivi psychothérapeutique, annonce son intention de tuer celle qu'il considère son amie de coeur. 
L'intervenant consulte deux psychiatres concernant la conduite à tenir, après quoi il informe les services policiers d'un risque éventuel de comportement homicide chez ce patient. En l'absence de tout comportement menaçant et devant les négations du suspect, les policiers le détiennent puis le libérent ne pouvant intervenir sur la foi de cette seule dénonciation.

Par ailleurs, la victime potentielle identifiée n'a pas été prévenue du risque qu'elle encourrait. Après sa libération, devant ce qu'il considère un bris de confidentialité, le client interrompt unilatéralement son suivi thérapeutique. Deux mois plus tard, il assassine la jeune femme.. Selon Tancredi et Weisstub (1986), cette position est en accord avec les principes éthiques de l'American Psychiatric Association : les psychiatres peuvent révéler des confidences afin de protéger la société d'un tort plus important.

Par comparaison, lorsqu'il est question de crimes passés, situation plus fréquente que celle du complot criminel. celle-ci est très peu abordée dans les écrits sur la question; d'où l'intérêt de notre présente démarche. Regardons maintenant comment la question de la confidentialité se situe au cœur de l’intervention clinique avec les délinquants.

\section{L'ACTIVITÉ CLINIQUE EN CRIMINOLOGIE}

L'expertise clinique de l'intervenant en criminologie s'articule autour de son désir de comprendre et d'aider son client à l'intérieur d'un mandat plus vaste de protection de la société. Les deux composantes sont essentielles : elles résument les fonctions de l'alliance thérapeutique et constituent le moteur même de l'entreprise évaluative ou d'intervention avec le client délinquant ou violent.

En règle générale, dans le cadre de ses activités cliniques auprès des délinquants, le criminologue doit porter plusieurs jugements d'ordre clinique. D'une part, il doit se prononcer à la fois sur la gravité (objective et subjective) du délit sanctionné, sur la dynamique de l'individu impliqué et sur les possibilités de récurrence de comportements déviants ou criminels. D'autre part, les objectifs cliniques à poursuivre dans l'immédiat et les moyens d'intervention spécifiques à utiliser s'inscrivent dans le sillage de l'évaluation, sans qu'il y ait nécessairement une nette distinction entre les deux types d'actions.

Quittant le domaine médical pour celui du contrôle de la déviance, le droit à la confidentialité caractérise une approche où l'on cherche à susciter l'adhésion du client à une définition commune du comportement problématique et aux différentes étapes de l'intervention visant à modifier ce comportement. En médecine comme en criminologie, la confidentialité demeure au service du client qui participe à un processus de changement personnel; agir autrement, par exemple, utiliser unilatéralement ou sans en informer le 
client des informations obtenues sous le couvert de la confidentialité, nourrirait un processus mensonger et sterile, dans lequel celui qui contrôle l'information triomphe sur son interlocuteur. Malheureusement ce dernier type de situation définit un type de relation présent dans plusieurs milieux de détention.

Dans un contexte clinique, il devient rapidement évident que la validité de l'analyse et de l'intervention criminologique repose sur l'exhaustivité des comportements problématiques répertoriés.

\section{L'IMPORTANCE DE CONNAÎTRE LES AGISSEMENTS RÉELS}

\section{La vérité d'un homme, c'est d'abord ce qu'il cache (Malraux).}

\section{Les agissements réels et les agissements officiels}

Officiellement, l'évaluation des agissements criminels s'effectue à partir de différentes sources : greffes criminelles des Cours, rapports d'événements policiers, etc; cependant le tableau de la délinquance adulte officielle. ainsi dépeint, ne représente qu'un pâle reflet de l'activité délinquante réelle.

Outre les consignations officielles, deux autres types d'approche permettent de mieux cerner l'activité criminelle globale. Premièrement, il est possible de tenter la quantification du nombre de personnes habitant une communauté donnée et ayant été victime(s) ou non d'acte(s) criminel(s), que ceux-ci aient été ou non dénoncés aux autorites: nous renvoyons ici aux études de victimisation.

À cet effet, une recherche effectuée en 1982, par le Groupe de Recherche du Solliciteur Général du Canada, ayant touché près de 61000 répondants âgés de plus de seize ans et habitant sept grands centres urbains canadiens, est révélatrice. Ainsi, selon la nature des incidents, seulement 29 à 70 p. cent de ceux-ci étaient signalés aux services policiers. Les délits les moins rapportés avaient trait au vol ou à une tentative de vol de biens personnels, tandis que ceux les plus régulièrement signalés concernaient le vol ou la tentative de vol d'un véhicule moteur.

Deuxièmement, en partant d'un point de vue différent, d'autres chercheurs se sont directement adressés à des échantillons de délinquants incarcérés afin de recueillir un dénombrement plus précis de leurs activités criminelles passées.

Utilisant cette seconde méthode, une étude effectuée aux États-Unis, en 1976, par Peterson et Braiker, a permis de compiler des résultats par rapport à une accusation ayant mené à l'incarcération des 624 détenus californiens qui constituaient l'échantillon. En effet au niveau de délits 
diversifiés tels l'homicide, le viol, le vol qualifié, le cambriolage, l'assaut, le vol d'auto et l'usage de faux, quatre résultats apparaissent :

1. la plupart des délinquants ayant commis un type particulier de crime n'ont pas été accusés de celui-ci ou reçu une sentence qui y était liée ;

2. un nombre substantiel de répondants affirment qu'ils commettaient peu fréquemment le délit pour lequel ils reçurent une sentence;

3. classifier les répondants selon le principal délit qui leur a mérité la peine, éclipse les différences entre les délinquants qui ont commis le même genre de délits ;

4. la plupart des délinquants se livrent à toute une gamme de délits; un groupe typique de 100 condamnés pour vol qualifié révélait avoir commis un total de 490 vols à main armée. 310 agressions contre des personnes, 720 cambriolages, 70 vols d'autos, 100 fraudes et 3400 ventes de drogues.

Pour notre propos, deux conclusions s'imposent donc; premièrement, tous les délits ne sont pas connus des instances officielles : certains ne font pas l'objet d'un signalement aux autorités, que ce soit par la victime ellemême ou par son entourage, et cela pour diverses raisons qui débordent le cadre du présent exposé. De plus, tous les signalements de délits ne se traduisent pas par des accusations, et ces dernières n'entraînent pas nécessairement des condamnations. Deuxièmement, les résultats obtenus par les questionnaires de délinquance révélée permettent de penser que, lorsqu'un individu est sentencé en fonction d'un délit particulier, ce dernier n'est ni son délit de prédilection, ni celui qu'il commet le plus fréquemment et que, enfin, ce délit ne révèle ni la gravité ni la diversité du comportement délictuel de l'individu.

Chose plus importante encore, au-delà des termes quantitatifs, le vécu du délinquant, avant pendant et après le passage à l'acte, échappe à ces instruments de cueillette primaires.

\section{Savoir pour intervenir}

Par l'intermédiaire de l'entrevue, l' «évalué» développe à la fois le sens de ses gestes et la responsabilité de leurs conséquences, et cela dans une perspective qui respecte tout autant l'objectivité des faits que l'intentionnalité des sujets, malgré les réflexes légitimateurs et victimisés qui sont les leurs et qui sont bien décrits dans les études cliniques. Cette attitude de départ présente pour le délinquant de nombreux «avantages» pour le maintien d'une estime de soi minimale. Elle permet de rejeter le blâme ou la responsabilité de ses actes sur autrui ou sur les circonstances, d'ignorer sa responsabilité personnelle et d'éviter de se demander quelles auraient pu être les autres façons de composer avec la réalité (Samenow et Yochelson, 1976). 
Le droit au secret professionnel pourrait, ici, faciliter le dévoilement du sujet et son ouverture vis-à-vis l'intervenant. Voilà qui augmenterait les chances de réussite de l'intervention, puisque la possibilité d'un traitement, à moyen et long terme, peut permettre l'amélioration des conditions psychologiques qui ont influencé l'accomplissement d'un crime et, du coup, réduire la récidive criminelle.

Cet accès à la confidentialité se fait présentement aux risques et périls du client. En effet, comme nous l'avons fait remarquer dans notre introduction, le droit au secret professionnel n'est pas enchâssé dans la charte canadienne des droits; de plus, tout intervenant qui reçoit des confidences en raison de sa profession (médecin, religieux, etc.) peut être contraint de témoigner en cour criminelle concernant les informations dont il est le dépositaire, la règle étant que l'intérêt supérieur de la justice doit primer sur le droit des individus.

Il est néanmoins possible pour un professionnel de demander à la Cour le privilège de se taire; il faudrait alors démontrer l'importance thérapeutique des confidences faites au cours du traitement et les conséquences graves que pourrait entraîner le bris du lien de confidence.

Simone Weil (1962) écrivait: «Un droit n'est pas efficace par luimême, mais seulement par l'obligation à laquelle il correspond. » Dans le cas du droit au secret professionnel, l'absence de reconnaissance au niveau de la «contraignabilité » des témoins en cour criminelle engendre des réticences tant chez les clients que chez les intervenants.

\section{LE SECRET PROFESSIONNEL ET LES RÉTICENCES LIÉES AU CLIENT}

\section{Toute révélation d'un secret est la faute de celui qui l'a confié (La Bruyère).}

Le développement d'une alliance entre le client et l'intervenant suppose l'existence d'une série de facteurs circonstanciels et d'attitudes présents avant et pendant le processus d'intervention criminologique. Ces facteurs exercent leur influence indépendamment de la qualité et de l'expérience des consultants impliqués et sans égard à la motivation, intrinsèque ou extrinsèque, de celui qui demande de l'aide. En ce sens c'est d'abord le cadre de pratique qui définit les conditions nécessaires à l'actualisation de l'alliance. Cette section analyse chacun de ces facteurs dans leurs rapports aux réticences des clients.

\section{Vocation et mandat du milieu}

Jusqu'à présent, nous n'avons soulevé la question du secret professionnel qu'à l'intérieur d'un contexte de relation de type dyade, entre l'intervenant et son client. Une telle limitation du débat ne doit pas éclipser le fait 
que ces interventions se tiennent dans un lieu ayant une vocation, une infrastructure, une organisation clinique et des objectifs propres. Ceux-ci s'incarnent dans les procédures et les règles, mais se vivent dans le climat et les relations intervenants/clients.

Si nous comparons le mandat social des milieux psychiatrique et pénitentiaire, nous constatons que celui-ci est semblable et vise avant tout à assurer la protection de la société.

Selon le document portant sur l'orientation générale du Service Correctionnel Canada (SCC) (1987), l'énoncé de mission se lit comme suit : «Partie intégrante du système de justice pénale, le SCC protège la sociêté et, pour ce faire, assure une surveillance humaine des détenus tout en les aidant à devenir des citoyens respectueux de la loi. »

Au niveau du SCC, l'atteinte de cet objectif s'actualise selon deux modes d'action complémentaires, soit : premièrement, par la garde physique des détenus puis, dans un second temps, lorsque (pour la grande majorité) ceux-ci bénéficient du privilège de terminer leur sentence en milieu communautaire, par le contrôle technique de la mobilité, des fréquentations et des occupations des surveillés.

Par contraste, le milieu médico-légal espère atteindre le même objectif final, mais par des moyens fort différents, objectif qui est d'offrir un lieu d'expertise, de recherche et de traitement destinés aux clientèles violentes. Cette intention se reflete évidemment au niveau de l'infrastructure et de l'organisation clinique du milieu : le climat, les règles, les relations se distinguent du milieu pénitentiaire et favorisent l'ouverture du délinquant.

Ainsi, dans le cas qui nous intéresse, les différences fondamentales existant entre la vocation et la philosophie de gestion et d'intervention des milieux de détention et hospitaliers conditionnent et rendent possible l'évaluation et l'intervention sophistiquées.

Ce travail de responsabilisation doit s'effectuer dans un contexte de confidentialité élevée. L'utilisation de l'expression «confidentialité élevée » s'explique par le fait qu'en ce domaine l'évocation du secret professionnel n'a qu'un rôle protecteur mitigé, cette raison n'ayant pas préséance (sauf pour les membres du Barreau) dans l'éventualité de poursuites judiciaires criminelles à l'endroit de notre client. L'individu est donc prévenu que la confidentialité promise ne relève pas de la totale confidentialité.

Ces dispositions légales et la nature clinique du travail que nous menons auprès des clients nous amènent à adopter une position de réceptivité, mais dont les limites sont claires. Il est essentiel d'assurer la confidentialité des propos qui nous sont révélés en entrevue puisque, sans elle, l'évaluation et le traitement des délinquants violents deviennent impossibles : 
comment cerner et contrer une problématique faite de violence et d'agressions sans accéder aux agissements réels et souvent non officiels de nos clients?

L'exploration des données en entrevue nous confronte évidemment à d'autres embûches quant à la validation des données recueillies. En effet, dans une perspective légitime d'autoprotection délinquante, le contrevenant a rationnellement tout intérêt à ce que ses agissements restent secrets, et cela aux yeux du plus grand nombre possible de personnes et aussi longtemps que possible, en particulier devant les gens qui détiennent un pouvoir découlant de l'exercice de fonctions judiciaires, para-judiciaires ou d'autorité entendue au sens large (parents, etc).

Ainsi le recours aux figures du milieu d'origine ou du milieu de vie extérieur ne permet généralement pas de valider les assertions incriminantes recueillies auprès du contrevenant lui-même. Ces constatations ont amené les équipes professionnelles travaillant avec ce type de clientèle violente à systématiquement communiquer aux sujets les réserves et les conditions entourant la période d'évaluation clinique.

Cette réceptivité comporte aussi le «risque» que le client mente; cependant ne pas courir ce risque équivaudrait, pour nous, à nous résigner à la pauvreté documentaire des statistiques officielles en matière de criminalité. Selon notre expérience, ce risque est apparu largement contrôlable par l'exploration systématique du récit du client. À un degré moindre, des confirmations indirectes par des pairs contribuent aussi à réduire 1'influence théoriquement attribuable à ce facteur.

Ces éléments apparaissent stimulants, sur le plan professionnel et clinique. car ils démontrent que le délinquant, lorsqu'il est approché d'un point de vue thérapeutique, dans un contexte d'aide disponible, peut se confier a l'intervenant clinique et rendre possible le début d'une entreprise ardue de changement personnel véritable.

Cette discussion soulève la question de l'efficacité des systèmes judiciaire et correctionnel. Ainsi, $s$ 'il est possible de comprendre rationnellement que la justice ne suive son cours moral que dans un nombre restreint de cas, comment ne pas se questionner sur les possibilités réelles du SCC d'atteindre les objectifs ambitieux de sa mission, lui qui évalue à un niveau officiel ceux qui lui sont confiés, faute de ne pas s'être donné les moyens d'établir un contexte clinique plus révélateur?

Rappelons, en terminant, que sur le plan déontologique, les révélations auto-accusatrices des clients ne sont recueillies qu'en fonction de la plus grande pertinence des interventions thérapeutiques ultérieures. 


\section{Confidentialité du dossier}

De par l'assujettissement des milieux hospitaliers à la loi provinciale sur l'accès à l'information, diverses mesures restreignent, $d$ 'une part, l'accès au dossier du client aux seuls professionnels de l'équipe traitante. D'autre part, cette même loi place le contrôle des demandes d'information et des autorisations de communiquer de l'information entre les mains du client concerné. Ainsi l'envoi de rapports d'expertise au SCC ou à la Commission Nationale des Libérations Conditionnelles (CNLC) relève de l'autorisation du client traité.

Au niveau du SCC, ce type de confidentialité n'existe pas. De plus le personnel du SCC et de la CNLC est soumis à l'obligation de transmettre au procureur général tout délit non officiel dont il pourrait être informé au cours d'une entrevue ou lors d'audiences menées avec des délinquants fédéraux.

Si le client est prêt à en assumer les risques, le contexte hospitalier permet. donc d'offrir une confidentialité élevée, tant au niveau de l'évaluation que du travail clinique à effectuer auprès de la clientèle concernée.

\section{Le sujet : désir de changement}

Une autre dimension importante tient à la nature de la motivation du client. L'alliance avec l'évaluateur et le désir d'un certain changement motivé, soit par le dégoût du milieu et du style de vie criminel, soit par la tension découlant des enjeux quotidiens de vie ou de mort, ou encore le constat d'une vie d'échecs personnel et social, etc. augmentent la probabilité que graduellement le délinquant se confie sans fard.

\section{Confiance en l'intervenant}

Afin d'éviter d'être placé ou de placer le client dans une situation légale délicate, il est important d'aviser au préalable l'évalué qu'il peut choisir de ne pas divulguer un certain nombre d'informations, par exemple l'identité des victimes, le lieu précis du délit, etc., l'importance pour nous étant de prendre connaissance du ou des scénario(s) prédélictuel(s) et délictuel(s) afin d'en identifier les éléments d'évaluation et d'intervention avec le client.

Ainsi l'invitation à tout dire, faite en début de processus évaluatif, entraîne des modulations différentes selon le contexte où elle se présente. Le cadre de travail décrit permet de le faire en mettant l'accent sur le désir de changer du client et le besoin pour ce faire de prendre ses responsabilités vis-à-vis de son passé.

\section{Attentes d'aide}

Certaines attitudes au cours de l'entrevue facilitent le développement essentiel d'une alliance client intervenant. Le milieu hospitalier procure un contexte où le client délinquant peut plus facilement croire en la présence 
d'aidants. Donc, il faut aussi que nos attitudes reflètent l'aide disponible en contrepartie de nos exigences d'honnêteté et d'effort responsable.

La première attitude est celle de la disponibilité. Il s'agit d'être prêt à consacrer le temps nécessaire à l'exploration et à l'évaluation du passé du client afin de s'assurer de la meilleure connaissance phénoménologique et chronologique possible du cas. Quantitativement le fait signifie plusieurs dizaines d'heures d'entrevue consacrêes à l'évaluation d'un seul client.

Les attitudes classiques, faites de respect et d'écoute du client, prennent une connotation active où la fermeté, l'exploration systématique et la confrontation mènent à un respect plus réel et réciproque. Le caractère direct de cette approche et l'exigence d'effort et d'honnêteté qui l'accompagnent produisent, dans la plupart des cas, un effet déstabilisateur marqué (Samenow et Yochelson, 1976). Ces auteurs soulignent a juste titre que les délinquants ont généralement à leur actif de nombreuses heures d'expérience de l'entrevue et qu'ils n'hésitent pas à utiliser la manipulation et la séduction. D'ailleurs, ils ont généralement réussi à obtenir ce qu'ils voulaient par ces pratiques (classification sécuritaire, recommandation d'élargissement de tout genre, etc.) et ce à un moindre coût en ce qui les concerne.

Nous avons identifié certains facteurs liês aux réticences des détenus lorsque vient le temps de révéler leurs activités criminelles réelles. Regardons maintenant quelle sera la contrepartie chez les intervenants recevant ce type de confidences.

\section{LE SECRET PROFESSIONNEL ET LES RÉTICENCES LIEESS AUX INTERVENANTS}

Un secret, ce n'est pas quelque chose qui ne se raconte pas. Mais c'est une chose qu'on se raconte à voix basse et séparément (Pagnol).

L'invitation à tout dire faite au début d'un processus évaluatif entraîne des interrogations diverses quant à notre engagement vis-à-vis de la société en tant que citoyen et professionnel spécialisé dans la délinquance. En voici quelques-unes.

\section{Le code de déontologie de l'APCQ et la confidentialité}

Au Québec, faute de statut défini par le code des professions, c'est l'Association professionnelle des criminologues du Québec (APCQ) qui élabore les normes d'exercice professionnel de ces intervenants.

Les normes de déontologie de l'APCQ sont claires concernant le secret professionnel : le criminologue, ses associés et employés doivent respecter le secret de tout renseignement de nature confidentielle obtenu dans l'exercice 
de leur profession. C'est la règle, sauf lorsque le client l'y autorise ou lorsque la loi l'ordonne; il faut aussi rappeler que le droit professionnel est de juridiction provinciale et est donc subordonné au droit criminel de nature fédérale.

Sur le plan éthique, il faut prévenir le client des conséquences possibles de révélations concernant des délits pour lesquels il n'a pas été poursuivi. Cette mise en garde prend la forme d'un engagement de respecter le secret professionnel absolu, sauf dans l'éventualité d'une assignation à témoigner devant une cour criminelle. Cette position s'appuie sur le principe de l'amélioration possible des conditions profondes ayant influencé l'émergence de la délinquance chez cet individu.

Par contre, une confidence qui aurait trait à la préparation d'un crime ne tombe pas sous le coup du secret professionnel ; dans ces cas, les autorités seraient alors saisies de l'affaire. Ceci nous amène à aborder la question du misprision.

\section{Le misprision}

Le common law anglais comporte la notion de misprision, soit l'omission de rapporter la connaissance d'un crime; cependant, ce concept n'existe ni dans le droit canadien ni dans les législations de la majorité des états américains.

Si nous le mentionnons ici, c'est que le misprision décrit le mieux la réalité légale du secret d'un délit inconnu des autorités judiciaires. En effet, il se distingue tant de la complicité après le fait (article 23 (1) du Code Criminel Canadien (CCC)) que de l'entrave à la justice (article 139 du CCC). L'absence de composante active ${ }^{3}$, visant à faire échapper quelqu'un à la justice le démarque du premier.

Par ailleurs, la formulation codifié du crime d'entrave à la justice porte tour à tour sur l'obtention d'avantages financiers touchant l'indemnisation ou le versement d'une caution, la prestation d'un témoignage ou l'intimidation des témoins et des jurés (paragraphes 1 et 3 ). Demeure la définition plus générale de l'entrave en tant que démarche visant à entraver, à détoumer ou à contrecarrer le cours de la justice. Ici, le caractère non su des confidences recueillies au cours du travail clinique rend, selon nous, ces propos incompatibles avec la condition préalable d'une procédure judiciaire existante ou projetée se rapportant au crime révélé.

Ce contexte juridique entraîne deux types de conséquences : d'une part, en s'intéressant à l'agissement délinquant non judiciarisé le praticien a

3. Appelbaum (1986) mentionne quelques exemples tirés de la jurisprudence américaine : destruction de preuves, hébergement d'un criminel, intimidation de témoins, etc. 
souvent l'impression de marcher sur une corde raide au risque d'y perdre une crédibilité, personnelle et professionnelle chèrement acquises.

D'autre part, bien qu'il n'existe pas d'obligation formelle de dénoncer tout délit non poursuivi dont on pourrait prendre connaissance au cours du traitement d'un client, il demeure qu'un certain nombre de situations peuvent remettre en question nos valeurs personnelles. Peu importe que ces situations soient prévues ou non au niveau de directives ou de politiques institutionnelles; ces instruments peuvent codifier la conduite à adopter, mais non le jugement moral qui l'accompagne.

Faudrait-il toujours privilégier la «confidentialité élevée» si l'on apprend qu'un autre a été condamné à la place du client qui s'accuse devant nous? Il est vrai que l'investigation du récit des clients ne se fait pas selon un mode policier et que, techniquement, les «aveux » reçus ne sont que des ouï-dires. Néanmoins devrait-on attendre du praticien qu'il évalue la portée statistique et scientifique du traitement offert tout en considérant du même souffle, ses valeurs personnelles et la «traitabilité » du client en question? Pour nous la question reste entière.

\section{Risques pour le récipiendaire des confidences}

La rubrique précédente portait sur les interrogations morales et légales se rattachant à la délinquance cachée. Il existe cependant un niveau de préoccupation hypothétique, dont les retombées ont un caractère à la fois plus concret et plus pertinent à la présente réflexion.

Le confident peut en venir à être perçu par le client comme une entrave au processus d'élargissement de son présent mandat d'incarcération, en plus de constituer une menace, encore plus grande, à l'endroit d'une nouvelle accusation potentielle. Voilà qui peut facilement définir un champ où s'exerceraient pressions, harcèlement et, ultimement, violence afin de préserver l'immunité de fait.

Pour prévenir le développement d'une telle situation, il nous semble primordial de «déprivatiser» le caractère de la confidence reçue et d'en informer celui qui s'est confié. Cela se fait d'abord au niveau de l'équipe traitante multidisciplinaire, puis, de l'organisme, en tant que responsable des décisions administratives concernant le client.

\section{Responsabilité civile et criminelle}

Sans être assujetti au misprision, le praticien demeure responsable de ses actes au sens civil et criminel. Ainsi l'usage de la confidentialité qui auráit causé un tort à une tierce partie ou constitué un acte de négligence criminelle pourrait-il faire l'objet de poursuites et de recours.

Une situation hypothétique est celle où d'un innocent, qui a reçu une sentence et est incarcéré pour un crime grave, et où le thérapeute refuse de dévoiler aux autorités l'identité (vérifiée) du coupable qui s'accuse devant lui. 


\title{
L'IMPORTANCE DU SECRET PROFESSIONNEL
}

\author{
Ce n'est pas toujours le pire \\ que les hommes cachent (Mauriac).
}

Évoquer l'éthique en rapport avec la pratique clinique en criminologie, c'est accepter d'aborder des sujets souvent complexes ou ambigus, qui peuvent, de surcroît, mener facilement à la controverse. Expliquons-nous : d'une part, tout clinicien, qu'il soit psychiatre, psychologue ou criminologue, véhicule ses valeurs personnelles dans son milieu de travail et lors de ses interventions auprès des clients.

D' autre part, le contexte de pratique est aussi à même de nous confronter aux valeurs de nos clients et de nos collègues, si ce n'est aussi aux dimensions éthiques liées à l'application de lois ou à des conflits de valeurs potentiels ou réels. Ce dernier aspect nous intéresse particulièrement, car le praticien se sent alors souvent obligé de choisir entre la compréhension et l'aide concrète à apporter à un client dans un contexte, plus abstrait, de protection de la société.

Si les problématiques éthiques foisonnent au sein de la pratique criminologique et rejoignent tous les criminologues dans leurs activités cliniques quotidiennes, paradoxalement, elles apparaissent souvent méconnues à la fois des criminologues eux-mêmes et des autres intervenants de la justice pénale. De plus, il nous faut constater qu'il existe très peu d'écrits sur ce sujet.

Le présent article visait à explorer et clarifier le champ éthique relatif au secret professionnel tel qu'il se définit par l'intervention clinique auprès des délinquants adultes faisant l'objet d'un terme d'emprisonnement de nature fédérale. L'analyse des conditions et des règles de pratique inhérentes à l'activité clinique en criminologie nous amène à constater que l'exercice du droit du client au secret professionnel se heurte à des limites d'ordre légal ou judiciaire et à des réticences de la part des interlocuteurs en présence.

En tant que criminologue, ces questions doivent nous faire réfléchir profondément sur le plan éthique et sur celui de l'administration de la justice pénale.

Est-il possible, aujourd'hui, de faire accepter au système judiciaire le principe voulant qu'il existe plusieurs façons de rendre justice? Que le fait d'utiliser le secret professionnel afin de favoriser un processus de changement chez un délinquant et, donc, de prévenir la récidive, ses coûts et ses souffrances, tant pour le délinquant que pour ses victimes, constituent une autre façon tout aussi morale de faire cuuvre de justice? 
Nous pensons que oui : la confidentialité absolue doit être accessible au client dans un contexte permettant de l'aider à s'amender de manière responsable et constructive. Cette vision est la seule qui respecte les assises du principe de bienfaisance qui a dominé la déontologie médicale et clinique jusqu'à aujourd'hui (Grunberg, 1990).

Contraindre des intervenants cliniques à agir comme témoins à charge à l'endroit de leurs (ex-)clients serait élever la délation au rang de profession.

\section{BIBLIOGRAPHIE}

APPELBAUM, Paul S. (1984), "Confidentiality in the forensic evaluation», International Journal of Law and Psychiatry, vol. 7, p. 285-300.

APPELBAUM, Paul S. (1986), MEISEL, Alan, «Therapists' obligations to report their patients' criminal acts ", Bulletin of the American Academy of Psychiatry and Law, vol. 14, n³, p. 221-230.

Association Professionnelle des Criminologues du Québec, Code de déntologie, juin 1986.

Charte des Droits et Libertés du Québec (art. 5 et 9).

Charte canadienne des Droits et Libertés (art. 1, 7, 8, 11d, 15 et 26).

DEL RIO Y, Victor Bernal (1980), « Psychiatric ethics », in Harold I. Kaplan et Benjamin J. Sadock (ed.), Comprehensive textbook of psychiatry, vol. 2, troisième édition.

DEL RIO Y, Victor Bemal (1985), « Psychiatric ethics and confidentiality », in Harold I. Kaplan et Benjamin J. Sadock (éd.), Comprehensive textbook of psychiatry, vol. 2, troisième édition.

DUFRESNES, Jacques (1962), Le procès $d u$ droit, page 71 , citation originale : Simone Weil, L'Enracinement, Paris, NRF, Collection Idées, page 9.

EICHELMAN, Burr, Daniel WIKLER et Anne HARTWIG (1984), «Ethics and psychiatric research : problems and justification », American Journal of Psychiatry, $141, \mathrm{n}^{\circ} 3, \mathrm{p} .400-405$.

FREEDMAN, Alfred M. (1980), « Confidentiality », in Harold I. Kaplan et Benjamin J. Sadock (éd.), Comprehensive textbook of psychiatry, vol. 2, troisième édition.

Gouvernement du Canada (1987), Le Service Correctionnel du Canada Orientation Générale 1987.

Gouvernement du Canada (1983), Le sondage canadien sur la victimisation en milieu urbain, bulletin 1 .

GRUNBERG, Frédéric (1990), «La doctrine du consentement libre et éclairé : ses fondements éthiques, juridiques et ses applications dans la recherche et la pratique de la psychiatrie $\%$, Revue canadienne de psychiatrie, vol. 57, p. 443-450.

MOORE, Robert A. (1978), «Ethics in the practice of psychiatry- origins, functions, model and enforcement $*$, American Journal of Psychiatry, 135, $\mathrm{n}^{\circ} 2$, p. 157-163. 
PETERSON, M.A., H.B. BRAIKER et S.M. POLICH (1981), Who commits crime?, Cambridge, Grunn and Han.

REDLICH, Fritz et Richard F. MOLLICA (1976), "Overview : ethical issues in contemporary psychiatry ", American Journal of Psychiatry, 133, $\mathrm{n}^{\circ} 2, \mathrm{p} .125-136$. YOCHELSON, S. et S. SAMENOW (1976), The criminal personnality, New York, J. Aronson,

TANCREDI, Laurence R. et David N. WEISSTUB (1986), "Law, psychiatry and morality : unpacking the muddled prolegomenon ", International Journal of Law and Psychiarry, 9, p. 1-38.

WINSLADE, William J. (1989), «Ethics in psychiatry », in Harold I. Kaplan et Benjamin J. Sadock (éd.), Comprehensive textbook of psychiatry, vol. 2, cinquième édition. 